\title{
BMJ Open Protocol for a pilot randomised controlled trial of mindfulness-based cognitive therapy in youth with inflammatory bowel disease and depression
}

\author{
Tatjana Ewais, ${ }^{1,2}$ Jake Begun, ${ }^{1,3}$ Maura Kenny, ${ }^{4,5}$ Kai-Hsiang Chuang, ${ }^{6}$ \\ Johanna Barclay, ${ }^{3}$ Karen Hay, ${ }^{7}$ Steve Kisely ${ }^{2,8}$
}

To cite: Ewais T, Begun J, Kenny M, et al. Protocol for a pilot randomised controlled trial of mindfulness-based cognitive therapy in youth with inflammatory bowel disease and depression. BMJ Open 2019;9:e025568. doi:10.1136/ bmjopen-2018-025568

- Prepublication history for this paper is available online. To view these files, please visit the journal online (http://dx.doi. org/10.1136/bmjopen-2018025568).

Received 22 July 2018 Revised 19 December 2018 Accepted 12 March 2019

Check for updates

(C) Author(s) (or their employer(s)) 2019. Re-use permitted under CC BY-NC. No commercial re-use. See rights and permissions. Published by BMJ.

For numbered affiliations see end of article.

Correspondence to

Dr Tatjana Ewais;

tatjanaewais@gmail.com

\section{ABSTRACT}

Introduction Inflammatory bowel disease (IBD) is a chronic autoinflammatory disease of the gastrointestinal tract with peak age of onset during adolescence and young adulthood. Adolescents and young adults (AYAs) with IBD experience higher depression rates compared with peers who are well or have other chronic conditions. Mindfulness-based interventions are of particular interest because of their potential to improve both the course of IBD and depression. Methods and analysis This study is a parallel design, single-blind, pilot randomised controlled trial (RCT) of mindfulness-based cognitive therapy (MBCT) in AYAs with IBD and depression. The trial aims to recruit 64 participants who will be randomly allocated to MBCT or treatment as usual. The primary outcome measure is the depression subscale score from the Depression, Anxiety and Stress Scale. Secondary outcomes include anxiety, stress, post-traumatic growth, IBD-related quality of life, illness knowledge, medication adherence, mindfulness, IBD activity, inflammatory markers, microbiome and brain neuroconnectivity changes. All outcomes other than neuroimaging will be collected at three time points: at baseline, at therapy completion and at 20 weeks. Neuroimaging will be conducted at baseline and at therapy completion. Mixed-effects linear and logistic regression modelling will be used to analyse continuous and dichotomous outcomes, respectively. Participants' experiences will be explored through focus groups, and thematic analysis will be used to generate relevant themes. Ethics and dissemination The protocol has been approved by the Mater Hospital Human Research Ethics Committee (HREC) and University of Queensland HREC. Trial findings will be published in peer-reviewed journals and will be presented at scientific conferences.

Trial registration number ACTRN12617000876392, U1111-1197-7370; Pre-results.

\section{INTRODUCTION}

Inflammatory bowel disease (IBD) is an immune-mediated condition characterised by chronic inflammation of the gastrointestinal tract and frequent extraintestinal symptoms. ${ }^{12}$ The peak age of onset is between

\section{Strengths and limitations of this study}

- This study will be the first randomised controlled trial (RCT) of mindfulness-based cognitive therapy in adolescents and young adults with inflammatory bowel disease (IBD), a cohort with a peak age of IBD onset.

- Outcome measures will include psychological measures, as well as inflammatory markers, disease activity and neuroimaging, and will potentially contribute to explaining the mindfulness intervention mechanisms of action.

- The results from this study will be used to inform the design and sample size calculation of a future large RCT.

- The study will be run as a pilot RCT because variability in the effect sizes of mindfulness interventions and unknown effect size in this population make it difficult to estimate the sample size accurately.

- The pilot nature of the study will enable assessment of the intervention feasibility in this cohort.

the ages of 15 and 29, thus interrupting a crucial developmental stage and impacting on relationships, education and employment opportunities. ${ }^{3}$ Adolescents and young adults (AYAs) with IBD experience depression rates that are three times higher than those in the general population and higher than depression rates in youth with other chronic diseases with reported rates of current depression of up to $25 \% .^{4-6}$ They frequently experience impaired health-related quality of life (QoL) and delayed social development compared with their peers. ${ }^{5}$

\section{Depression in IBD}

The relationship between depression and IBD is bidirectional in that IBD can both cause and exacerbate depression, while depression can precede the onset of IBD, as 
well as worsen its course and prognosis. A recent systematic review has shown that depression increases the risk of exacerbation and recurrence of symptoms of IBD. ${ }^{7}$ There are a number of ways that depression and IBD interact, including common risk factors, decrease in treatment adherence and health-promoting behaviours, and shared underlying immune system abnormalities. ${ }^{8}$ Despite the impact of depression on the course of IBD, research into treatments for depression in these patients has been sparse and limited by poor methodological quality. Antidepressant medications have shown promise in treating depression and anxiety associated with IBD as well as improving IBD symptoms, although further studies with better methodological quality are needed. ${ }^{9}$ Psychosocial interventions with the most evidence include cognitive behavioural therapy, hypnosis and mindfulness-based interventions. ${ }^{10}{ }^{11}$ However, the evidence for psychological therapies in IBD has been mixed, with best results seen in improvement of depression and QoL and non-significant improvements seen in IBD symptoms and disease activity. ${ }^{10} 11$

\section{Mindfulness interventions and mindfulness-based cognitive therapy}

Mindfulness is a particular state of awareness described as 'paying attention in a particular way: on purpose, in the present moment and non-judgmentally'. ${ }^{12}$ Mindfulness-based interventions are therapeutic interventions based on core mindfulness principles and containing various mindfulness practices. They have been proven effective in treating depression and anxiety in diverse clinical populations, including those with chronic illness. ${ }^{13} 14$ Furthermore, by improving depression and anxiety, which are known predictors of treatment non-adherence and poor engagement, ${ }^{15}$ mindfulness interventions can lead to improved treatment adherence, healthcare empowerment and improvement in the course of chronic illness.

Research has shown that mindfulness programmes are associated with changes in neural structure and function in brain regions important for improved emotional well-being (experience dependent neuroplasticity), ${ }^{16}$ suggesting possible neural mechanisms for mindfulness-attenuating symptoms associated with mental health disorders. ${ }^{17-19} \mathrm{~A}$ recent systematic review of the impact of mindfulness interventions on the immune system parameters indicated that mindfulness can also modify immune activity, with the authors concluding that 'mindfulness meditation may be salutogenic for immune system dynamics, but additional work is needed to examine these effects' (p. 1). ${ }^{20}$

Accumulating evidence from mindfulness studies in IBD populations suggests that these interventions are associated with reduced depression and anxiety levels and improved QoL in individuals with IBD. ${ }^{1021}$ Mindfulness-based cognitive therapy (MBCT), an 8-week manualised group programme combining mindfulness with cognitive therapy and delivered in weekly sessions of 2 hours' duration, ${ }^{22}$ has been shown to be effective for the treatment of depression, ${ }^{23}$ as well as for the prevention of depressive relapse, ${ }^{24}$ and has recently been modified for use in IBD. ${ }^{25}$ Recent studies suggest that MBCT may be particularly helpful in individuals with significant baseline depressive symptoms, with a larger treatment effect in this cohort, and further reduction of depressive symptoms over time. ${ }^{2326}$

To date, there have been only a few published studies exploring the potential utility of mindfulness interventions in individuals with IBD and only one randomised controlled trial (RCT) of MBCT, conducted in adults with IBD, with the mean age of 49 . This RCT reported a significant improvement in depression and trait anxiety and small, not statistically significant, improvements in IBD activity and QoL ${ }^{25}$ however, no biological markers were measured to detect potential changes in inflammation and brain function. To further investigate the effectiveness and feasibility of this novel therapy in young adult patients with IBD and to elucidate its mechanism of action, we will conduct a pilot RCT of MBCT and measure changes in psychological outcomes as well as inflammatory biomarkers and brain connectivity following the intervention.

\section{Hypotheses}

1. MBCT intervention will be feasible and acceptable as indicated by sessions attendance, completion of home practices, recruitment and attrition rates, and participants' MBCT group experiences.

2. MBCT intervention will result in significantly improved depression scores post intervention.

3. MBCT will decrease anxiety, stress and maladaptive coping, and increase QoL, post-traumatic growth (PTG), mindfulness, medication adherence, self-efficacy, healthcare empowerment, illness knowledge and perceptions.

4. MBCT intervention will be associated with decreased inflammatory burden and improved microbiome.

5. MBCT will change the structural and functional connectivity in default mode network (medial prefrontal cortex and posterior cingulate cortex) and executive control network (dorsolateral prefrontal cortex and frontoparietal regions).

\section{Study aims}

Using 64 volunteer individuals (aged 16-29) with IBD and depression, we will explore the feasibility and benefits of an IBD-focused, developmentally informed MBCT programme on depressive symptoms in AYAs living with IBD and comorbid depression. Secondary aims are to explore the potential benefits of the MBCT on additional outcomes, including

- Anxiety, stress, coping, QoL, PTG, mindfulness, medication adherence, self-efficacy, healthcare empowerment, and illness knowledge and perceptions.

- Biological markers of IBD. 
- Brain structure and function using functional MRI (fMRI) performed on a subgroup of 16 intervention and 16 control subjects.

Specific aims related to the pilot nature of the study are the following:

- To determine the feasibility of the mindfulness programme by measuring the participants' attendance, compliance with home mindfulness practices, recruitment and attrition rates, and MBCT group experiences.

- To adapt the MBCT intervention to young adult patients with IBD while measuring the intervention delivery fidelity.

- To use the findings to elucidate mindfulness mechanisms of action.

- To use data from this pilot RCT to inform the design and sample size calculation for a future large RCT.

\section{METHODS AND ANALYSIS}

\section{Study design}

This is a parallel design, single-blind, pilot RCT of an adapted MBCT programme for AYAs with IBD and depression versus treatment as usual (TAU). The design adheres to the Consolidated Standards of Reporting Trials (CONSORT) statement recommendations and CONSORT extensions for pilot trials. ${ }^{27} 28$ We followed the Standard Protocol Items for Randomised Trials (SPIRIT) statement recommendations in writing the trial protocol. ${ }^{29}{ }^{30}$ The flow of the participants through the study is shown in figure 1.

\section{Recruitment strategies}

Participants will be recruited from the IBD outpatient services at Mater Young Adult Health Centre in Brisbane and surrounding IBD clinics via a specified referral pathway. Recruitment will be initiated during outpatient appointments, and those who are interested will meet with the research assistant who will explain the study in more detail, conduct the initial screening and obtain informed consent. Screening questionnaires will be made available in electronic and paper form, and email and text reminders will be used to minimise the burden to participants and facilitate recruitment. After participants consent, they will attend an interview to confirm the clinical diagnosis of depression and other inclusion criteria and ensure that there are no exclusion criteria to participation. The intake interview will be an unstructured clinical interview conducted by the principal investigator, who is a psychiatrist by training and who will assess whether participants meet the criteria for a major depressive disorder as defined by the Diagnostic and Statistical Manual of Mental Disorders. ${ }^{31}$ Recruitment and enrolment will be conducted in groups of 16-24 participants with 8-12 participants per MBCT group, which will suit the clinical nature of the group and provide optimal support.

\section{Participant selection}

Since this is a study of MBCT in AYAs with IBD and depression, main eligibility criteria will include age, IBD diagnosis and depression diagnosis. Complete inclusion and exclusion criteria are listed in boxes 1 and 2 .

\section{Randomisation}

Randomisation will be conducted by an off-site statistician not involved in patient recruitment or assessment, using random allocation software, after participants have provided written consent. The statistician will be given de-identified patient details generated by a researcher not involved in the recruitment, assessment or treatment. The statistician will generate the allocation schedule using the software and return it to the same researcher who will then match the participants' codes to their details and reveal the allocation.

To ensure a balanced composition between groups in terms of age, gender and depression levels, stratified randomisation will be performed by user-written RALLOC module ${ }^{32}$ in the Stata statistical package. ${ }^{33}$ Patients will be stratified by two categories for gender, age and depression scores. Within each stratum, patients will be allocated to MBCT or TAU. Because of limited resources, only $50 \%$ of the participants $(n=32)$ will be randomly allocated to have neuroimaging (fMRIs) using the same statistical package. Participants will be recruited and allocated in groups of 16-24 participants in order to accommodate the clinical nature of the group and to provide sufficient numbers. Participants and those delivering the intervention will not be blinded due to the nature of the intervention; however, research personnel involved in assessing the outcomes and data analysis will be blinded.

\section{Adapted MBCT Programme}

The MBCT programme, which will be used in this trial, closely follows the original MBCT curriculum designed by Segal, Williams and Teasdale,$^{22}$ and consists of eight weekly sessions of approximately 2 hours' duration. The adaptations will introduce IBD-specific education about stress and 'gut-brain axis' and developmentally informed and IBD-adapted mindfulness practices. The programme will be delivered by an experienced mindfulness teacher who is a mental health clinician with teachers' level training in Mindfulness-based Stress Reduction and MBCT, and who has delivered several mindfulness courses and maintained personal practice for 5 years. Supervision during the study will be provided by a senior MBCT supervisor who is a founding member of the Mindfulness Training Institute of Australia. Fidelity of the delivered intervention will be appraised by assessing video recordings of the sessions by the study MBCT supervisor and discussing the adherence to session content and delivery in supervision. Feasibility of the programme will be assessed from sessions attendance, home practices completion rates, recruitment and attrition rates, and participants' MBCT group experiences, which will be explored through postintervention focus groups. We will conduct three focus groups with six 


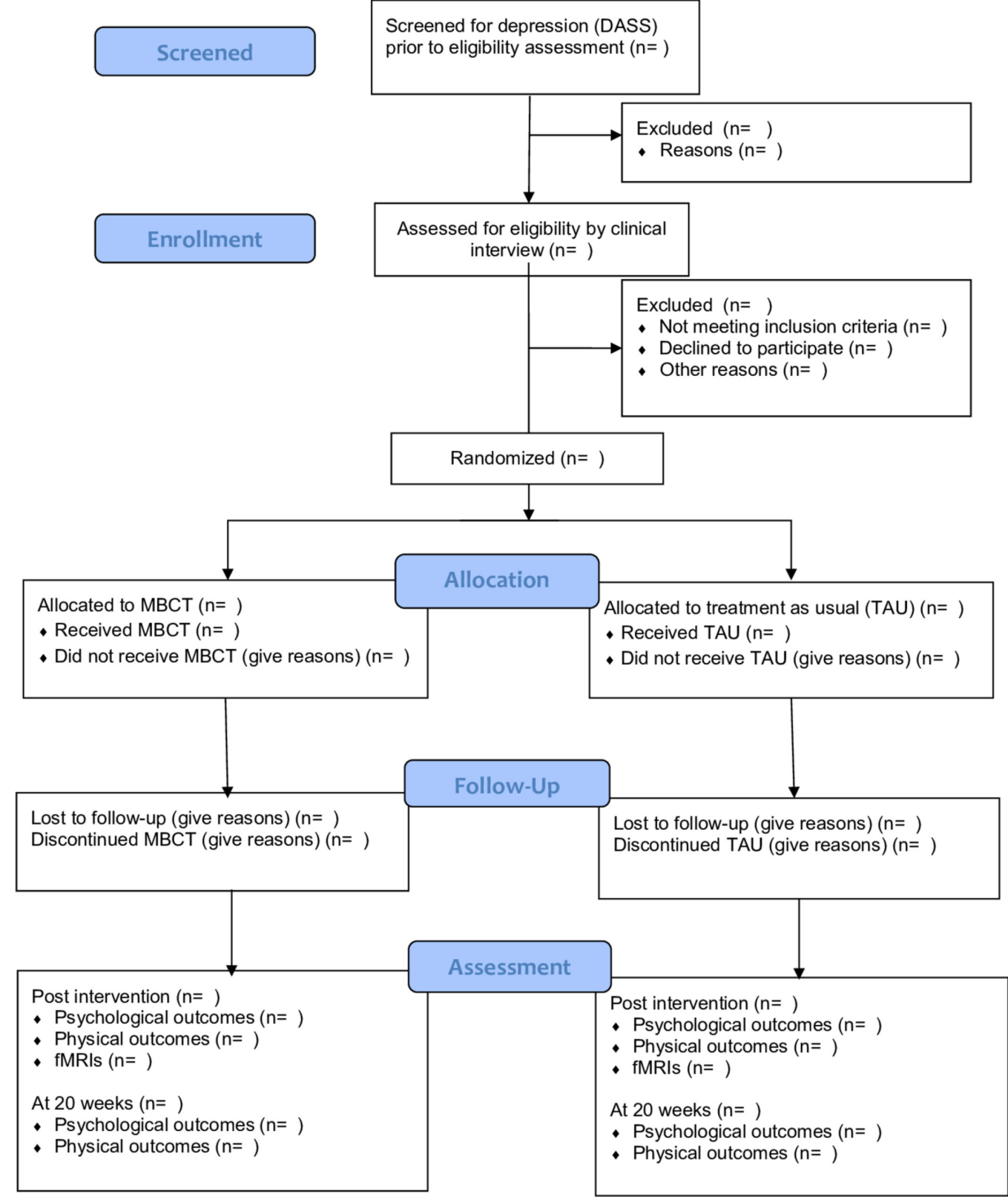

Figure 1 CONSORT extension for pilot and feasibility trials flow diagram showing the flow of the participants through the study. CONSORT, Consolidated Standards of Reporting Trials; DASS, Depression, Anxiety and Stress Scale; fMRI, functional MRI; MBCT, mindfulness-based cognitive therapy; TAU, treatment as usual .

to eight participants in each group in keeping with recommendations for running a minimum of three to four groups to enable conducting across groups analysis of patterns and themes, ${ }^{34}$ and recommended group size for clinical populations. Consecutive sampling will be used by inviting all participants who completed MBCT groups (five out of eight sessions as this is the accepted benchmark), via email and phone, until required numbers are recruited.

Participants will be given the adapted MBCT manual and a USB with guided mindfulness practices, and encouraged to engage in mindfulness practices for $45 \mathrm{~min}$ per day. They will be asked to record their home mindfulness practices daily in the home practice record form.

\section{Data collection and analysis}

Participants will be asked to complete questionnaires and provide blood and stool samples at baseline (week 0 ), on completion of the 8-week therapy programme or TAU (week 8) and at 20 weeks after baseline. The data gained from the research will be stored in a reidentifiable format with linkage of the data to patient details only able to be performed by the study research assistant.

The research assistant will complete a screening and recruitment sheet with details of all patients approached regarding the study, enrolled and excluded at each stage of the recruitment process, and reasons for this. The MBCT group facilitator will complete weekly attendance sheets to enable the calculation of attendance and 


\section{Box 1 Inclusion criteria}

Y Young adult (aged 16-29).

- Able to verbally communicate and write in English.

- Able to give informed consent (for youth aged $16-18$, both the participant and their parent/guardian).

- Confirmed diagnosis of IBD.

- Confirmed clinical diagnosis of depression and Depression, Anxiety and Stress Scale depression subscale score of 10 and above.

- Attending the IBD outpatient clinic at the Mater Young Adult Health Centre Brisbane.

- Have access to internet-enabled computer.

- Able to do light exercise because this programme involves a mindful movement component.

- Able to commit to attend the 8 weekly sessions of 2 hours' duration.

- Able to commit to do home practice of up to $45 \mathrm{~min}$ per day over the 8 weeks of the study as this is a requirement of the programme.

- No change in antidepressant medication (dose or type) within 3 months of trial onset.

attrition rates in the intervention group. These data will inform the recruitment strategies for a future large RCT.

This is a repeated measures study, so mixed-effects linear regression modelling will be used to assess the effect of the intervention on continuous, approximately normally distributed outcome measures (depression, anxiety and stress scores, and inflammation levels). Stratification variables (gender, age group and baseline depression level) will be controlled for in the analyses. In addition, a logistic mixed-effects model will be used to assess the effect of the intervention on clinical remission (binary outcome, defined as Depression, Anxiety and Stress Scale [DASS] depression subscale score less than 10). We will conduct an intention-to-treat analysis as well as a per-protocol

\section{Box 2 Exclusion criteria}

Individuals who do not have conversational or written English.

- Individuals who may have limited or no capacity for self-care.

- Individuals who have no depression or have extremely severe levels of depression (eg, associated with acute suicidal thoughts and/ or delusional beliefs and other psychotic symptoms).

- Individuals with major mental illness other than depression as its treatment and symptoms could interfere with their ability to participate in the programme (eg, current psychotic symptoms and post-traumatic stress disorder).

- Individuals with a history of or current alcohol or drug dependency.

- Individuals scheduled for major surgery in the next 3 months as this would impact on their ability to attend the programme for its entire duration.

- Individuals who have been started on antidepressants or have changed their antidepressant dose within 3 months of the study onset.

- Individuals enrolled or participating in another psychological therapy study or pharmacological study within the last 6 months or intending to participate during this study duration.

- Exacerbation of IBD symptoms (flare) as this will make it difficult for participants to attend weekly sessions and complete home practice. analysis. Finally, the proportions lost to follow-up will be reported and compared between groups.

In terms of the qualitative data, focus groups will be audio-recorded, transcribed and analysed using thematic analysis, a widely used and flexible qualitative research method for identification, analysis and reporting of key themes and patterns within the data. ${ }^{35}$ An inductive thematic analysis approach will be used, as it fits our aim of exploring AYA experiences of the MBCT group and the novelty of the topic being investigated. The data will be analysed iteratively and inductively until coherent and distinct themes develop, and thematic saturation is achieved, as indicated by no new codes and themes emerging.

\section{Safety considerations}

There have been no reports of significant adverse side effects of MBCT in literature with a recent meta-analysis that canvassed all completed MBCT studies for adverse events, confirming that no MBCT-related adverse events were reported.$^{26}$ Safety reporting will be done in keeping with Good Clinical Practice guidelines, including the reporting of all adverse events and serious adverse events (SAEs), both related and unrelated to the study, throughout the study duration. Participants will be screened and carefully selected for their suitability for the programme. They will be asked about any perceived adverse events, whether or not these may be related to the mindfulness intervention, and this information will be recorded in the trial database.

All SAEs will be reported within 24 hours to the Mater and University of Queensland (UQ) Human Research Ethics Committees (HRECs). All aspects of safety, recruitment and quality control of the data will be monitored by the Mater and UQ HRECs, and there will be no separate Data Safety and Monitoring Board involved. Participants will continue to receive standard medical and psychosocial care both during and after the conclusion of the trial.

\section{Sample size calculation}

Based on researcher experience, we expect 64 patients to be available to participate in the study, with a drop-out rate of $15 \%$. This sample size provides $80 \%$ power to identify large (Cohen's $\mathrm{D}=0.8$ ) effects. However, given the variability of the effect sizes for mindfulness studies, we have decided to run this study as a pilot RCT to assess the intervention's feasibility and effectiveness, as well as to provide data for establishing sample size for a definitive large RCT. The proposed sample size is in keeping with guidelines, which recommend a sample size of at least 40 for pilot studies and for effectiveness studies, 30 participants per arm of the study. ${ }^{35}$

\section{Outcomes}

All outcome tools chosen for the study are valid, reliable and routinely used in the setting of IBD and chronic illness. The primary outcome measure will be the depression subscale score from the DASS. ${ }^{36}$ 
DASS is a self-report instrument comprising three scales designed to measure three negative emotional sates of depression, anxiety and stress. All three scales of the DASS have been shown to have high internal consistency and meaningful discriminations in a variety of clinical and research settings. The short version, DASS 21, will be used in the study as it has shown validity comparable with the long version and will pose lesser burden to complete for the participants.

Secondary outcomes will include anxiety and stress scores on DASS, coping patterns on the brief version of coping inventory (Brief COPE), IBD-related QoL (Short Inflammatory Bowel Disease Quality of Life Questionnaire [SIBDQ]), PTG scores on Post-traumatic Growth Index (PTGI), mindfulness (Five Facet Mindfulness Questionnaire [FFMQ]), medication adherence, self-efficacy and healthcare empowerment (Medication Adherence Questionnaire [MAQ], Self-efficacy for Appropriate Medication Use Scale [SEAMS] and Healthcare Empowerment Inventory [HCEI] ), illness knowledge and perception (Crohn's and Colitis Knowledge Score [CCKNOW] and Illness Perception Questionnaire [IPQ]). SIBDQ is a 10-item questionnaire that is a validated and reliable instrument for assessing health-related QoL in patients with IBD patients. ${ }^{37}$ PTGI is a 21-item questionnaire measuring growth and positive changes following traumatic events ${ }^{38}$ and has been identified as a validated screening tool in individuals with IBD. ${ }^{39}$ The FFMQ is a validated screening tool assessing mindfulness as a multifaceted construct. ${ }^{40}$ The CCKNOW, Brief IPQ and Brief COPE are short, validated questionnaires for assessing illness knowledge, perception and coping, commonly used in clinical practice and research in the IBD population. ${ }^{41-43}$ Morisky 4-Item MAQ, SEAMS and HCEI are also brief, validated and reliable self-report questionnaires used to assess medication adherence, self-efficacy and healthcare empowerment, in IBD population. ${ }^{44-46}$

Secondary outcomes will also include IBD activity questionnaires (Simple Clinical Colitis Activity Index [SCCAI] and Harvey-Bradshaw Index [HBI]); biological markers of inflammation, both systemic (C reactive protein [CRP], erythrocyte sedimentation rate [ESR] and interleukin [IL]-6) and IBD-specific (faecal calprotectin and small intestinal ultrasound); microbiome analysis as it reflects IBD activity; and neuroconnectivity changes (fMRIs). We are measuring inflammation markers and neuroconnectivity as research has shown that mindfulness practices can attenuate inflammation and improve neuroconnectivity, and changes in these parameters combined with changes in psychological outcomes could contribute to explaining mindfulness mechanism of action.

\section{Patient and public involvement}

Patients' views informed the study design and they were obtained during the study conception from Mater Consumer Youth Consultancy Group members who provided feedback regarding the study. Participants' group experiences and burden of the intervention will be assessed through focus group interviews and informal feedback. Patients will not be involved in the recruitment and conduct of the study. Results from this study will be disseminated to each participant as a lay summary of the findings and through presentations at consumer consultancy forums.

\section{DISCUSSION}

This study is the first RCT of a mindfulness-based intervention in AYAs with IBD and depression, a vulnerable group of young patients with a high burden of IBD and significant long-term impairments in multiple areas of functioning. In keeping with research evidence of a larger treatment effect of MBCT in individuals with significant baseline depressive symptoms, the study will recruit participants with clinical diagnosis of depression.

The study will be using an adapted MBCT programme for AYAs with IBD that, if proven feasible, may become integrated into the early intervention pathways for IBD. The intervention could contribute to altering disease progression, reduce disabling symptoms and improve long-term outcomes in this group of patients.

The study will explore the feasibility of the adapted MBCT programme by recording sessions attendance, completion rates of home mindfulness practices, recruitment and attrition rates, as well as participants' MBCT group experiences assessed through focus groups. It will assess the effectiveness of the MBCT programme by measuring its impact on various psychological and physical outcomes of IBD and depression, including biological markers of inflammation and brain connectivity. The study has the potential to validate this type of intervention, improve patient outcomes in young IBD sufferers and translate this approach to other chronic illness patient groups. The inclusion of biologic measures of disease activity and functional neuroimaging will allow additional insights into the relationship between IBD and depression, and mindfulness' mechanism of action.

\section{Study status}

An amendment to the protocol was approved in January 2018 (version 3, 18 January 2018), extending the upper age range to 29 and replacing the Hospital Anxiety and Depression Scale with the PTGI. Additional amendment to protocol was approved in October 2018 (version 4, 11 October 2018), removing the upper limit of the DASS from the inclusion criteria. The study is currently in the recruitment stage, with completion expected by the end of 2019.

\footnotetext{
Author affiliations

${ }^{1}$ Mater Young Adult Health Centre, Mater Misericordiae Brisbane Ltd, South Brisbane, Queensland, Australia

${ }^{2}$ Faculty of Medicine, University of Queensland, Brisbane, Queensland, Australia ${ }^{3}$ Mater Research Institute-UQ, University of Queensland, Brisbane, Queensland, Australia

${ }^{4}$ Faculty of Health and Medical Sciences, University of Adelaide, Adelaide, South Australia, Australia

${ }^{5}$ Teaching Faculty, Mindfulness Training Institute, Sydney, New South Wales, Australia
} 
${ }^{6}$ Queensland Brain Institute, University of Queensland, Brisbane, Queensland, Australia

${ }^{7}$ Statistics Unit, QIMR Berghofer Medical Research Institute, Herston, Queensland, Australia

${ }^{8}$ Princess Alexandra Hospital, Metro South Hospital and Health Service, Woolloongabba, Queensland, Australia

Acknowledgements The authors would like to thank the Mater Youth Consultancy Group members for their input in study conception and design, Simon Knowles for his advice on trial methodology, Anne Tremellen for her advice on translating the protocol into a successful ethics application and Merilyn Tefay for her assistance in adapting the mindfulness-based cognitive therapy manual.

Contributors Study protocol was conceived and designed by TE and critically revised by SK, JBe, MK, KH, KC and JBa. Drafting of the paper was completed by TE. All authors edited the manuscript.

Funding This work was supported by a project grant from the Brain Injured Children's Aftercare Recovery Endeavours (BICARE).

\section{Competing interests None declared.}

Ethics approval The study was approved by the ethics committees of the Mater Hospital and The University of Queensland in June 2017.

Provenance and peer review Not commissioned; externally peer reviewed.

Open access This is an open access article distributed in accordance with the Creative Commons Attribution Non Commercial (CC BY-NC 4.0) license, which permits others to distribute, remix, adapt, build upon this work non-commercially, and license their derivative works on different terms, provided the original work is properly cited, appropriate credit is given, any changes made indicated, and the use is non-commercial. See: http://creativecommons.org/licenses/by-nc/4.0/.

\section{REFERENCES}

1. Graham DB, Xavier RJ. From genetics of inflammatory bowel disease towards mechanistic insights. Trends Immunol 2013;34:371-8.

2. Kumar V, Wijmenga C, Xavier RJ. Genetics of immune-mediated disorders: from genome-wide association to molecular mechanism. Curr Opin Immunol 2014;31:51-7.

3. Johnston RD, Logan RF. What is the peak age for onset of IBD? Inflamm Bowel Dis 2008;14(S2 Suppl):S4-S5.

4. Greenley RN, Hommel KA, Nebel J, et al. A meta-analytic review of the psychosocial adjustment of youth with inflammatory bowe disease. J Pediatr Psychol 2010;35:857-69.

5. Fuller-Thomson E, Sulman J. Depression and inflammatory bowel disease: findings from two nationally representative Canadian surveys. Inflamm Bowel Dis 2006;12:697-707.

6. Neuendorf R, Harding A, Stello N, et al. Depression and anxiety in patients with inflammatory bowel disease: a systematic review. $J$ Psychosom Res 2016;87:70-80.

7. Mikocka-Walus A, Pittet V, Rossel JB, et al. Symptoms of depression and anxiety are independently associated with clinical recurrence of inflammatory bowel disease. Clin Gastroenterol Hepatol 2016;14:829-35

8. Berk M, Williams LJ, Jacka FN, et al. So depression is an inflammatory disease, but where does the inflammation come from? BMC Medicine 2013;11.

9. Tarricone I, Regazzi MG, Bonucci G, et al. Prevalence and effectiveness of psychiatric treatments for patients with IBD: A systematic literature review. J Psychosom Res 2017;101:68-95.

10. Ballou S, Keefer L. Psychological interventions for irritable bowel syndrome and inflammatory bowel diseases. Clin Trans/ Gastroenterol 2017;8:e214.

11. Tarricone I, Regazzi MG, Bonucci G, et al. Prevalence and effectiveness of psychiatric treatments for patients with IBD: a systematic literature review. J Psychosom Res 2017;101:68.

12. Kabat-Zinn J. Wherever you go, there you are. New York: Hyperion, 1994

13. Hofmann SG, Sawyer AT, Witt AA, et al. The effect of mindfulnessbased therapy on anxiety and depression: A meta-analytic review. $J$ Consult Clin Psychol 2010;78:169-83.

14. Abbott RA, Whear R, Rodgers LR, et al. Effectiveness of mindfulness-based stress reduction and mindfulness based cognitive therapy in vascular disease: A systematic review and meta-analysis of randomised controlled trials. J Psychosom Res 2014;76:341-51.

15. World Health Organization. Adherence to long-term therapies: evidence for action. Geneva: World Health Organization, 2003.
16. Lazar SW, Kerr CE, Wasserman $\mathrm{RH}$, et al. Meditation experience is associated with increased cortical thickness. Neuroreport 2005;16:1893-7.

17. Luders E, Clark K, Narr KL, et al. Enhanced brain connectivity in long-term meditation practitioners. Neuroimage 2011;57:1308-16.

18. Scheibner HJ, Bogler $\mathrm{C}$, Gleich $\mathrm{T}$, et al. Internal and external attention and the default mode network. Neuroimage 2017;148(C):p. 381-389.

19. Taren AA, Gianaros PJ, Greco CM, et al. Mindfulness meditation training and executive control network resting state functional connectivity: a randomized controlled trial. Psychosom Med 2017;79:674-83.

20. Black DS, Slavich GM. Mindfulness meditation and the immune system: a systematic review of randomized controlled trials. Ann N Y Acad Sci 2016;1373:13-24.

21. Neilson K, Ftanou M, Monshat K, et al. A Controlled Study of a Group Mindfulness Intervention for Individuals Living With Inflammatory Bowel Disease. Inflamm Bowel Dis 2016;22:694-701.

22. Segal ZV, Williams JMG, Teasdale JD. Mindfulness-based cognitive therapy for depression. 2nd edn: The Guilford Press, 2013.

23. van Aalderen JR, Donders AR, Peffer K, et al. Long-term outcome of mindfulness-based cognitive therapy in recurrently depressed patients with and without a depressive episode at baseline. Depress Anxiety 2015;32:563-9.

24. Kuyken W, Byford S, Taylor RS, et al. Mindfulness-based cognitive therapy to prevent relapse in recurrent depression. J Consult Clin Psychol 2008;76:966-78.

25. Schoultz M, Atherton I, Watson A. Mindfulness-based cognitive therapy for inflammatory bowel disease patients: findings from an exploratory pilot randomised controlled trial. Trials 2015;16:379.

26. Kuyken W, Warren FC, Taylor RS, et al. Efficacy of mindfulnessbased cognitive therapy in prevention of depressive relapse: an individual patient data meta-analysis from randomized trials. JAMA Psychiatry 2016;73:565-74

27. Schulz KF, Altman DG, Moher D. CONSORT 2010 Statement: updated guidelines for reporting parallel group randomised trials. BMJ 2010;340:698:c332.

28. Moher D, Hopewell S, Schulz KF, et al. CONSORT 2010 Explanation and Elaboration: Updated guidelines for reporting parallel group randomised trials. J Clin Epidemiol 2010;63:e1-e37.

29. Chan AW, Tetzlaff JM, Altman DG, et al. SPIRIT 2013 statement: defining standard protocol items for clinical trials. Ann Intern Med 2013;158:200.

30. Chan AW, Tetzlaff JM, Gøtzsche PC, et al. SPIRIT 2013 explanation and elaboration: guidance for protocols of clinical trials. BMJ 2013;346:e7586

31. American Psychiatric Association. Diagnostic and statistical manual of mental disorders: DSM-5 T/ American Psychiatric Association. 5th ed. Washington, DC: American Psychiatric Pub, 2013.

32. Ryan P. "RALLOC: Stata module to design randomized controlled trials, " Statistical Software Components S319901: Boston College Department of Economics, 2018.

33. StataCorp. Stata statistical software: release 15. College Station, TX: StataCorp LLC, 2017.

34. Barbour R, Groups DF. Doing focus groups, ed. RosalineBarbour. United Kingdom, London: SAGE Publications Ltd, 2007.

35. Hertzog MA. Considerations in determining sample size for pilot studies. Res Nurs Health 2008;31:180-91.

36. Lovibond PF, Lovibond SH. The structure of negative emotional states: comparison of the Depression Anxiety Stress Scales (DASS) with the Beck Depression and Anxiety Inventories. Behav Res Ther 1995;33:335-43.

37. Irvine EJ, Zhou Q, Thompson AK. The Short Inflammatory Bowel Disease Questionnaire: a quality of life instrument for community physicians managing inflammatory bowel disease. CCRPT Investigators. Canadian Crohn's Relapse Prevention Trial. Am J Gastroenterol 1996;91:1571.

38. Tedeschi RG, Calhoun LG. The Posttraumatic Growth Inventory: measuring the positive legacy of trauma. J Trauma Stress 1996:9:455-71.

39. Purc-Stephenson RJ. The Posttraumatic Growth Inventory: factor structure and invariance among persons with chronic diseases. Rehabil Psychol 2014;59:10-18.

40. Baer RA, Smith GT, Hopkins J, et al. Using self-report assessment methods to explore facets of mindfulness. Assessment 2006;13:27-45.

41. Eaden JA, Abrams K, Mayberry JF. The Crohn's and Colitis Knowledge Score: a test for measuring patient knowledge in inflammatory bowel disease. Am J Gastroenterol 1999;94:3560-6.

42. Broadbent E, Petrie KJ, Main J, et al. The brief illness perception questionnaire. J Psychosom Res 2006;60:631-7. 
43. Carver CS. You want to measure coping but your protocol's too long: consider the brief COPE. Int J Behav Med 1997;4:92-100.

44. Morisky DE, Green LW, Levine DM. Concurrent and predictive validity of a self-reported measure of medication adherence. Med Care 1986;24:67-74.

45. Risser J, Jacobson TA, Kripalani S. Development and psychometric evaluation of the Self-efficacy for Appropriate Medication Use Scale
(SEAMS) in low-literacy patients with chronic disease. J Nurs Meas 2007:15:203-19.

46. Johnson MO, Rose CD, Dilworth SE, et al. Advances in the conceptualization and measurement of Health Care Empowerment: development and validation of the Health Care Empowerment inventory. PLoS One 2012;7. 